\title{
Strategic Design of Catalytic Lysine-Targeting Reversible Covalent BCR-ABL Inhibitors
}

David Quach, ${ }^{[a][b]}$ Guanghui Tang, ${ }^{[c]}$ Jothi Anantharajan, ${ }^{[b]}$ Nithya Baburajendran, ${ }^{[b]}$ Anders Poulsen, ${ }^{[b]}$ John L. K. Wee, ${ }^{[b]}$ Priya Retna, ${ }^{[b]}$ Rong Li, ${ }^{[b]}$ Boping Liu, ${ }^{[b]}$ Doris H. Y. Tee, ${ }^{[b]}$ Perlyn Z. Kwek, ${ }^{[b]}$ Joma K. Joy, ${ }^{[b]}$ Wan-Qi Yang, ${ }^{[d]}$ Chong-Jing Zhang, ${ }^{, d]}$ Klement Foo, ${ }^{*}{ }^{[b]}$ Thomas $\mathrm{H}$. Keller, ${ }^{*}$ Shao Q. Yao*[a][c]

\footnotetext{
[a] Dr. D. Quach, Prof. Dr. S. Q. Yao

NUS Graduate School for Integrative Sciences and Engineering, 21 Lower Kent Ridge, University Hall, Tan China Tuan Wing, \#04-02, Singapore 119077 E-mail: chmyaosq@nus.edu.sg

[b] Dr. D. Quach, Dr. J. Anantharajan, Dr. N. Baburajendran, Dr. A. Poulsen, J. L. K. Wee, P. Retna, R. Li, B. Liu, D. H. Y. Tee, P. Z. Kwek, Dr. J. K. Joy, Dr. K. Foo, Dr. T. H. Keller

Experimental Drug Development Centre, 10 Biopolis Road, Chromos, \#05-01, Singapore 138670

E-mail: thkeller@eddc.a-star.edu.sg, klementfoo@gmail.com

[c] Dr. G. Tang, Prof. Dr. S. Q. Yao

Department of Chemistry, National University of Singapore, Singapore 117543

[d] W-.Q. Yang, Dr. C-.J. Zhang

State Key Laboratory of Bioactive Substances and Functions of Natural Medicines and Beijing Key Laboratory of Active Substances Discovery and Druggability Evaluation, Institute of Materia Medica Peking Union Medical College and Chinese Academy of Medical Sciences, Beijing, China, 100050

Supporting information for this article is given via a link at the end of the document.
}

\begin{abstract}
Targeted covalent inhibitors have re-emerged as validated drugs to overcome acquired resistance in cancer treatment. Herein, by using a carbonyl boronic acid warhead, we report the structure-based design of BCR-ABL inhibitors via reversible covalent targeting of the catalytic lysine with improved single-digit nanomolar potency against both wild-type and mutant $A B L$ kinases, especially $A B L^{\top 3151}$ bearing the gatekeeper residue mutation. We show that, by using techniques including mass spectrometry, time-dependent biochemical assays and X-ray crystallography, the evolutionarily conserved lysine can be targeted selectively. Furthermore, we show that the selectivity depends largely on molecular recognition of the non-covalent pharmacophore in this class of inhibitors, probably due to the moderate reactivity of the warhead. We report the first co-crystal structures of covalent inhibitor-ABL kinase domain complexes, providing insights into the interaction of this warhead with the catalytic lysine. We also employed label-free mass spectrometry to evaluate potential off-targets of our compounds at proteomewide level in different cancer cell lines.
\end{abstract}

\section{Introduction}

Chronic myeloid leukemia (CML) arises from a genetic abnormality in human chromosome 22, which is unusually short and defective because of the reciprocal translocation of genetic material from chromosome 9. ${ }^{[1]}$ Gene expression leads to the formation of a constitutively active BCR-ABL1 kinase, which aberrantly activates multiple signaling pathways that bring about uncontrollable cell growth and differentiation. [2] Despite the clinical success of ATPcompetitive inhibitors, ${ }^{[3]}$ a significant number of patients

\section{A. Reported lysine modification by Taunton[22]}<smiles>C=CCNC(=O)c1cc(Nc2cc(CC)[nH]n2)nc(N2CCN(Cc3ccc(S(=O)(=O)NCCCC)cc3)CC2)n1</smiles>

B. Iminoboronate mechanism [30]

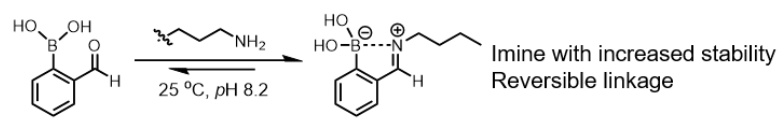

C. In-gel fluorescence labeling of BSA

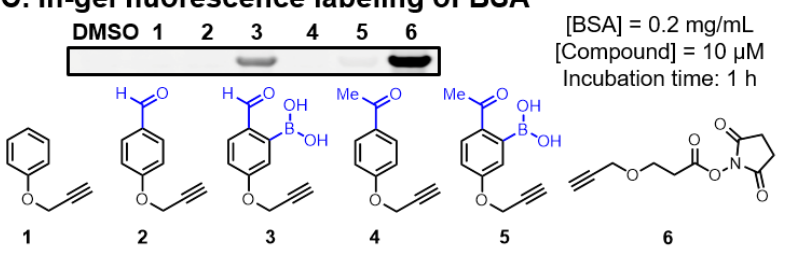

D. This work<smiles>COc1ccccc1-c1c[nH]c2ncc(-c3cncc(C(=O)N(C)C)c3)cc12</smiles>
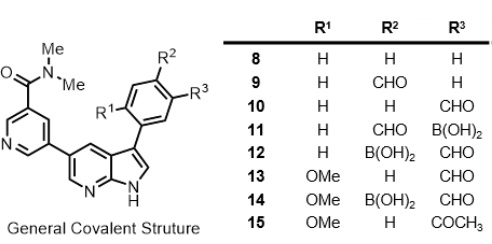

Figure 1. (A) A previously reported sulfonyl fluoride probe for covalent lysine labeling of kinases in live cells. ${ }^{[22]}$ (B) Lysine modification based on the formation of a stable iminoboronate with 2-formylbenzene boronic acid. ${ }^{[30]}$ (C) Model study of various lysine-targeting probes by using bovine serum albumin (BSA). (D) Structure-based design of BCR-ABL inhibitors targeting the catalytic lysine K271, based on a previously reported inhibitor PPY-A (7). ${ }^{[32]}$

have suffered from relapse due to drug resistance, which can arise from point mutations that severely reduce the effect of such inhibitors. ${ }^{[4,5]}$ An important mutant in $\mathrm{CML}$ is $\mathrm{BCR}-\mathrm{ABL}^{\mathrm{T} 315 \mathrm{I}}$ which can only be inhibited by ponatinib (Iclusig). ${ }^{[6]}$ Targeted covalent 


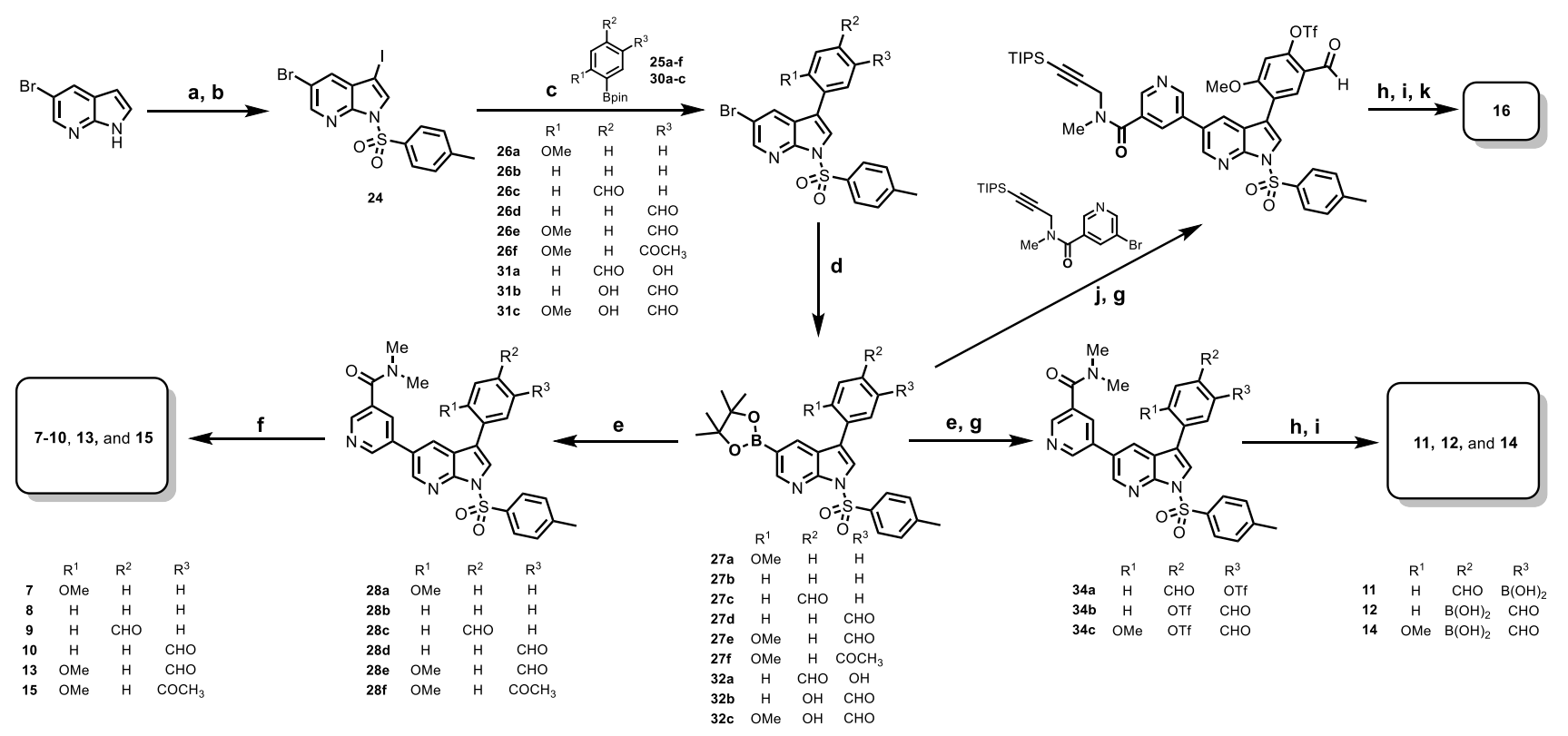

Scheme 1. Synthesis of key inhibitors used in this work. (a) NIS, acetone, rt, $1 \mathrm{~h}, 98 \%$; (b) TsCl, $\mathrm{NaH}, \mathrm{THF}, 0{ }^{\circ} \mathrm{C}, 3 \mathrm{~h}, 96 \%$; (c) $\mathrm{Pd}(\mathrm{dppf}) \mathrm{Cl}$, $\mathrm{K}_{2} \mathrm{CO}_{3}$, 1,4-dioxane $/ \mathrm{H}_{2} \mathrm{O}(9: 1), 70{ }^{\circ} \mathrm{C}, 7 \mathrm{~h}, 60-80 \%$; (d) $\mathrm{B}_{2} \mathrm{pin}_{2}, \mathrm{Pd}(\mathrm{dppf}) \mathrm{Cl}_{2}, \mathrm{KOAc}, 1,4$-dioxane, $100{ }^{\circ} \mathrm{C}, 7 \mathrm{~h}, 70-90 \%$; (e) 5-bromo- $\mathrm{N}, \mathrm{N}$ dimethylnicotinamide, $\mathrm{Pd}$ (dppf) $\mathrm{Cl}_{2}, \mathrm{~K}_{2} \mathrm{CO}_{3}, 1,4$-dioxane/ $\mathrm{H}_{2} \mathrm{O}(9: 1), 95{ }^{\circ} \mathrm{C}, 7 \mathrm{~h}, 70-80 \%$; (f) $\mathrm{LiOH}, \mathrm{MeOH} /$ dioxane/ $\mathrm{H}_{2} \mathrm{O}$ (2:2:1), rt, $1 \mathrm{~h}$, up to 90\%; (g) $\mathrm{CF}_{3} \mathrm{SO}_{2} \mathrm{Cl}, \mathrm{K}_{2} \mathrm{CO}_{3}$, DMF, rt, 2-4 h, 80-90\%; (h) $\mathrm{B}_{2} \mathrm{pin}_{2}$, $\mathrm{Pd}$ (dppf) $\mathrm{Cl}_{2}, \mathrm{KOAc}$, 1,4-dioxane, $100{ }^{\circ} \mathrm{C}, 7 \mathrm{~h}$; (i) $\mathrm{Cs}_{2} \mathrm{CO}_{3}, \mathrm{THF} / \mathrm{MeOH}^{2}: 1$ ), $40-50{ }^{\circ} \mathrm{C}, 1 \mathrm{~h}, 10-20 \%$ (2-step yields from (h) and (i)); (j) 5-bromo- $N$-methyl- $N$-(3-(triisopropylsilyl)prop-2-yn-1-yl)nicotinamide, $\mathrm{Pd}(\mathrm{dppf}) \mathrm{Cl}{ }_{2}$, $\mathrm{K}_{2} \mathrm{CO}_{3}, 1,4$-dioxane $/ \mathrm{H}_{2} \mathrm{O}(9: 1), 95{ }^{\circ} \mathrm{C}, 7 \mathrm{~h}, 80-90 \%$; (k) TBAF, THF/ $\mathrm{H}_{2} \mathrm{O}$, rt, 2.5\% (3-step yield from (h), (i) and (k)).

inhibitors offer advantages such as greater potency and prolonged duration of action over non-covalent inhibitors, and they have reemerged in recent years as demonstrated by the clinical success of, for example, osimertinib. ${ }^{7-111]}$ The standard strategy to design irreversible kinase inhibitors uses Michael acceptors to target poorly conserved cysteine residues near the active site of kinases. ${ }^{[12]}$ This was thought to provide a measure of selectivity; however, Cravatt and coworkers have demonstrated that even carefully designed cysteine-targeting covalent inhibitors have off-target effects. ${ }^{[13]}$ Furthermore, resistance mechanisms including cysteine point mutations (EGFRC797s and BTKC481S) often render cysteine-targeting covalent drugs ineffective. ${ }^{[11]}$ To our knowledge, no covalent drugs against any of the known BCR-ABL mutants have been reported up to date due to a lack of targetable cysteine residues. ${ }^{[14]}$

Since the catalytic lysine residue is essential for the enzymatic activity of all protein kinases and therefore, considered less prone to mutation, ${ }^{[15,16]}$ we have been interested to study this evolutionarily conserved residue in the ATP pocket with the aim to produce covalent kinase inhibitors as an alternative strategy in drug design. Many non-selective lysine-modifying probes, including the use of sulfonyl fluorides and activated esters, have been reported.[17-23] Taunton and coworkers were the first who reported sulfonyl fluoride probes for lysine-targeting in kinases (Figure 1A).[22] Campos et al later identified lysine-targeting kinase inhibitors that used an activated ester.[23] Since cellular toxicity is a major concern for both tool compounds and drug candidates, many research groups aspire to tune the reactivity of covalent warheads.[24-28] For example, Taunton and co-workers used electron-deficient Michael acceptors for the design of cysteine-targeting reversible covalent kinase inhibitors. ${ }^{[29]}$ Iminoboronates have recently been shown to reversibly but covalently modify amino groups in proteins;[30] however, this chemistry, to the best of our knowledge, has not been used to develop covalent kinase inhibitors (Figure 1B). ${ }^{[31]}$ Our aim was therefore to design reversible, covalent inhibitors targeting the catalytic lysine residue in kinases as a general approach to combat drug resistance. We report herein the first successful examples of lysine-targeting reversible covalent kinase inhibitors based on iminoboronate chemistry (Figure $1 C / D)$; our results showed that such compounds possessed potent and long-lasting inhibition against BCR-ABL wild type and mutants.

\section{Results and Discussion}

At the outset, a mechanistic study was carried out to compare the relative reactivity of model probes $\mathbf{3}$ and $\mathbf{5}$ with an NHS probe (6) and other controls by using BSA as reference (Figure 1C); removal of either carbonyl or boronic acid (or both; e.g. 1, 2 and 4) caused complete abolishment in fluorescent labeling of BSA in $\mathbf{3}$ and $\mathbf{5}$. Between $\mathbf{3}$ and $\mathbf{5}$, the former consistently labeled BSA more strongly, indicating the aldehyde was more reactive than the ketone. As expected, both $\mathbf{3}$ and $\mathbf{5}$ produced much weaker fluorescence labeling of BSA compared to $\mathbf{6}$, suggesting iminoboronates have attenuated lysine reactivity compared to the highly 
reactive NHS-based irreversible lysine modifier. ${ }^{[18]}$ We next designed suitable iminoboronate-containing BCR$A B L$ kinase inhibitors (Figure 1D). Molecular modeling studies were first carried out by incorporating a carbonyl boronic acid warhead into a previously reported $A B L 1$ inhibitor PPY-A (7) (PDB ID: 2QOH; Figure S1);[32] results showed that introducing the required functionality would not disrupt the binding to the protein, and with the distance between the proposed carbonyl boronic acid and the highly flexible catalytic lysine K271 being $3.5 \AA$, formation of an iminoboronate in the kinase/inhibitor complex was indeed possible.

A library of analogs was synthesized in order to establish structure-activity relationship (Figures 1D and S2, Scheme 1). The synthesis of a common intermediate $\mathbf{2 4}$ was done in two steps via iodination and tosylation of 5-bromoazaindole. 7-10 were obtained in four steps via Suzuki-Miyaura coupling reactions involving $\mathbf{2 4}$ to generate 26a-d, which underwent Miyaura borylation that led to 27a-d. The SuzukiMiyaura cross coupling reaction was performed at a lower temperature of $60^{\circ} \mathrm{C}$ in order to chemoselectively differentiate $\mathrm{Br}$ and I. 27a-d then underwent a second
Suzuki-Miyaura coupling reaction with 5-bromo- $N, N$ dimethylnicotinamide followed by subsequent removal of tosyl group with $\mathrm{LiOH}$, leading to the formation of the desired inhibitors. The synthesis of carbonyl boronic acid inhibitors 11 and 12 occured via a different route in which an additional step involving the conversion of $\mathrm{OH}$ to OTf, led to intermediates $34 \mathrm{a}$ and $34 \mathrm{~b} ; \mathrm{CF}_{3} \mathrm{SO}_{2} \mathrm{Cl}$ and $\mathrm{K}_{2} \mathrm{CO}_{3}$ were shown to be the optimal choice. Other electrophiles such as $\mathrm{PhNTf}_{2}$ and $\left(\mathrm{CF}_{3} \mathrm{SO}_{2}\right)_{2} \mathrm{O}$ in the presence of bases such as triethylamine, $\mathrm{NaH}$ and pyridine did not work well, however, despite heating. 34a and 34b underwent borylation and subsequent deprotection of the tosyl group by using $\mathrm{Cs}_{2} \mathrm{CO}_{3}$ was carried out to generate covalent inhibitors 11 and 12 (Supporting Information).

By using a mobility shift assay based on Caliper's microfluidics capillary electrophoresis, the $\mathrm{IC}_{50}$ value of PPY-A (7) against wildtype ABL was determined to be $2 \mathrm{nM}$ (Figure S2). Removal of the o-methoxy group reduced the potency by 20 folds (compound 8 ). Introduction of $m$-aldehyde (compound 10), however, improved the $\mathrm{IC}_{50}$ to $8 \mathrm{nM}$. Shifting the aldehyde functional group to the para position was not tolerated
A
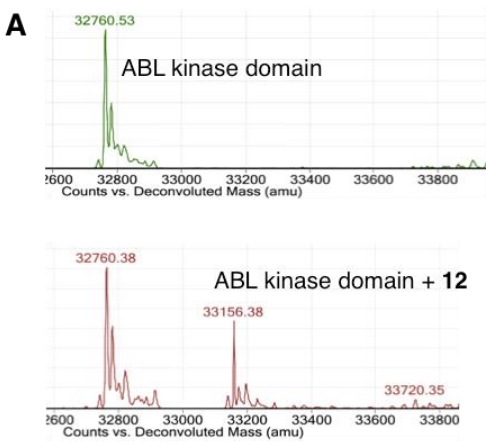

C

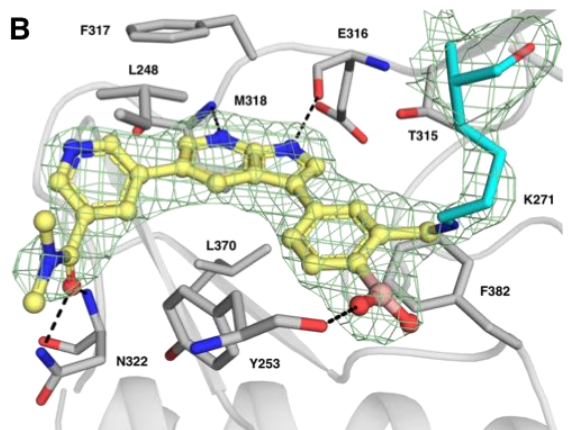

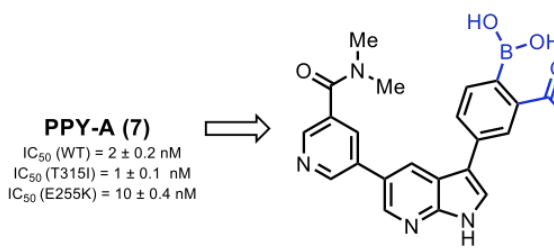
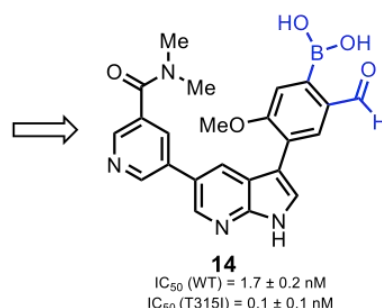

$\begin{aligned} I_{50}(\mathrm{~T} 3151) & =0.1 \pm 0.1 \mathrm{nM} \\ \mathrm{IC}_{50}(\mathrm{E} 255 \mathrm{~K}) & =0.5 \pm 0.03 \mathrm{nM}\end{aligned}$

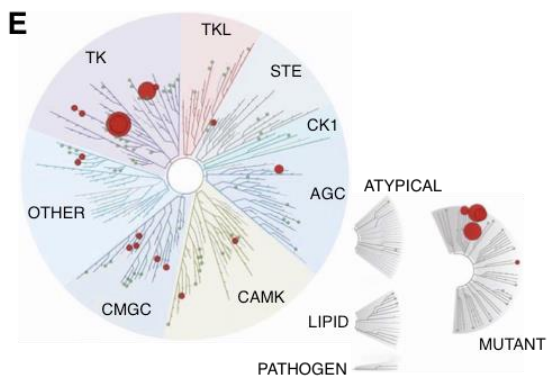

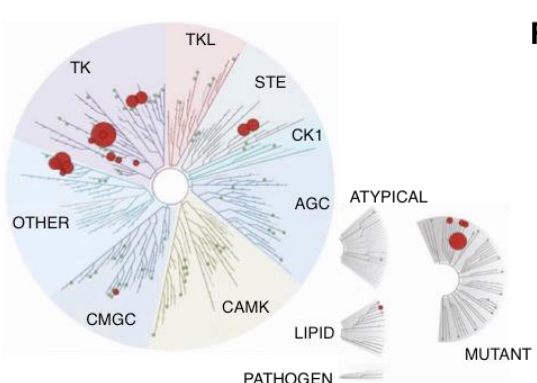

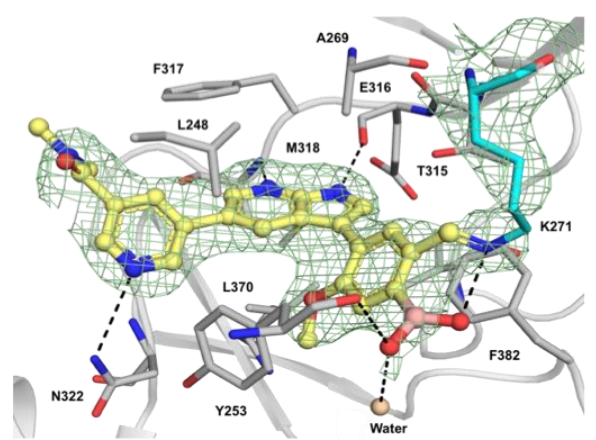

D

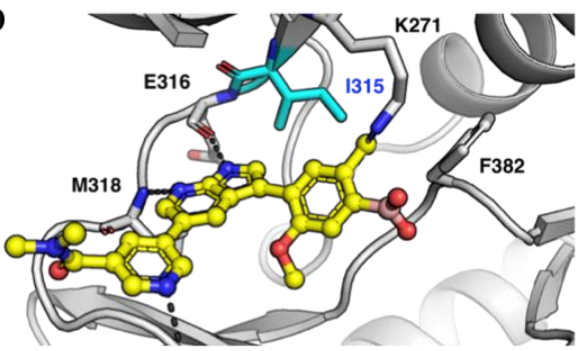

$\mathbf{F}$

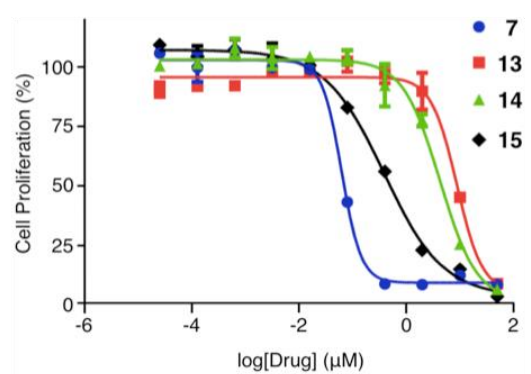

Figure 2. (A) Mass spectrometric analysis of the ABL kinase domain-12 complex. (B) Cocrystal structures of the ABL kinase domain with 12 (left) and 14 (right) showing the composite omit map $\left(2 \mathrm{~F}_{\mathrm{o}}-\mathrm{F}_{\mathrm{c}}\right)$ contoured to $1 \sigma$ in green mesh. The front loop was removed for better visibility. (C) Comparison of ABL inhibition (WT and mutants) of PPY-A (7), 12 and 14 after 6 or 12 h. (D) Modeled structure of ABL ${ }^{\text {T315l }}$ kinase domain14 complex based on the obtained cocrystal structure of ABL ${ }^{W T}$ kinase domain-14 complex. (E) Dendrograms showing Kinome Scan ${ }^{\text {TM }}$ of 7 (left) and 14 (right) at $1000 \mathrm{nM}$ against 90 different kinases. (F) Anti-proliferative activity of 7, 13, 14 and 15 against K562 cells determined by CellTiter-Glo® viability assay. 
(compound 9). Introduction of a boronic acid functionality led to inhibitors $\mathbf{1 1}$ and 12. As expected of covalent inhibitors, the enzyme inhibition of compound 12 improved from $83 \mathrm{nM}(\mathrm{T}=0 \mathrm{~h})$ to $5 \mathrm{nM}(\mathrm{T}=12 \mathrm{~h})$ as the incubation time was increased (Figure S3A). Compound $\mathbf{1 0}$ did not show time-dependence of the $\mathrm{IC}_{50}$, suggesting that the presence of the boronic acid in 12 may have led to the formation of an iminoboronate. ${ }^{[33]}$

To determine whether 12 was selective towards the catalytic lysine in $A B L$, mass spectrometric analysis was performed. MALDI-TOF analysis suggested that a single lysine-modified covalent adduct was formed with an observed $\mathrm{m} / \mathrm{z}$ of $33156.38 \mathrm{Da}$ (Figure $2 \mathrm{~A}$; compared to calculated mass of $33156.59 \mathrm{Da})$. The reaction did not reach completion regardless of the concentration (up to $1 \mathrm{mM}$ ) and incubation time (up to $24 \mathrm{~h}$ ) of $\mathbf{1 2}$, indicating that an equilibrium was established. ${ }^{[30,31]}$ Since the OMe group in 7 was important for $A B L$ inhibition, the same functionality was added to $\mathbf{1 2}$, providing compound 14 which was synthesized via a similar route (Scheme 1, Figure S6C). As expected, 14 showed time-dependent $\mathrm{IC}_{50}$ values against $\mathrm{ABL}$ from $13 \mathrm{nM}$ at $0 \mathrm{~h}$ to $1.7 \mathrm{nM}$ after $12 \mathrm{~h}$ (Figure 2C); further testing of 7 and 14 against two ABL mutants indicated that 14 was about ten-fold more potent against both $A B L^{T 315 I}$ and $A B L^{E 255 K}$ than 7, and at the same time also showed time-dependent inhibition against both mutants (Figures S3C and S3D). In particular, 14 demonstrated an improved potency from $25 \mathrm{nM}(\mathrm{T}=0 \mathrm{~h}$ ) to $0.1 \mathrm{nM}$ ( $\mathrm{T}$ $=6 \mathrm{~h}$ ) against $A B L^{\top 315 \mathrm{I}}$ (250-fold improvement) and from $43 \mathrm{nM}(\mathrm{T}=0 \mathrm{~h})$ to $0.5 \mathrm{nM}(\mathrm{T}=12 \mathrm{~h})$ against $A B L^{E 255 K}$ (100-fold improvement). The effect of point mutation on restricting access to the binding pocket or stabilizing certain protein conformations has been shown to adversely affect drug binding which tends to favor only a very specific target conformation; [10] however, our examples showed that targeting the catalytic lysine residues gave an advantage in this context.

The X-ray cocrystal structures of 12 and 14 with the ABL kinase domain (229-510) were solved up to $2.7 \AA$ and $2.3 \AA$ resolution, respectively, giving more insights into how this particular warhead interacts with the catalytic lysine residue (Figure 2B; PDB IDs: 7CC2 and 7DT2); the continuous electron density from $\mathrm{K} 271$ in the $A B L$ kinase domain to 12 and 14 suggests that the imine product was formed. We did not however observe the formation of the expected dative bond between the imine nitrogen and the boron atom. In fact, the lone pair of the imine appeared to be orientated away from the boron atom in both crystal structures. One of the reasons could be due to the vast structural difference between the macromolecule-inhibitor complex and small molecules given the tight binding pocket of a protein target (our data ${ }^{11} \mathrm{~B}$ NMR showed that an iminoboronate could be captured for small molecules, Figure S14). Since the biochemical assays and the MALDI-TOF analysis suggested that the boronic acid plays an important role in the formation of the adduct, we propose that the obtained cocrystal structures had successfully captured the key intermediate during the formation of the iminoboronates. Given the fact that 14 was able to potently inhibit $A B L^{\top 315 l}$ while imatinib and second-generation kinase inhibitors such as nilotinib, dasatinib failed to do so, ${ }^{[32]}$ we next rationalized this observation by using the newly obtained structural data (Figure 2D); by building a modeled structural complex of 14 and $A B L$ kinase domain in which the T315 residue was artificially changed to 1315 , we observed no steric clash upon inhibitor binding. Imatinib, nilotinib and dasatinib possess crucial elongated structures that are extended to the back cleft of the ATP binding pocket in $A B L$, and $T 315 \mathrm{I}$ point mutation was expected to restrict access to the hydrophobic region at the rear of this pocket. ${ }^{[32]}$ This is not the case for 14 which does not have any substituent that occupies this pocket. Finally, 14 demonstrated better biochemical activity than that of 7 due to apparent covalent modification, ${ }^{[34]}$ and the incorporation of both boronic acid and aldehyde did not appear to cause any steric clash with the 1315 residue.

The kinetic parameters of inactivation were next determined to better understand the non-bonded interaction of 12 and 14 with the kinase (Figures S4);[34] both compounds showed similar $k_{\text {inact }}$ values. while 14 had a smaller $\mathrm{K}_{\mathrm{I}}$ compared to that of 12 , thus confirming that the critical role of OMe in $\mathbf{1 4}$ for increased affinity between the inhibitor and the ABL kinase domain.

One of the potential concerns for covalent inhibitors like 14, which target a key amino acid in the active site of kinases, is the selectivity. We therefore carried out Kinome Scan ${ }^{\mathrm{TM}}$ with a panel of $\sim 100$ protein kinases (Figure 2E); results showed highly similar interaction maps for compounds 7 (left) and 14 (right), suggesting that the degree of compound selectivity largely depended on the initial step of molecular recognition presumably due to the moderately reactive 2-carbonyl boronic acid warhead in 14. The conclusion was further strengthened by the selectivity scores (Table S4). To evaluate the anti-proliferative activity of this class of inhibitors, compounds 7 and 14 , as well as reference compounds 13 and 15 (boronic acid-free versions of 14; see Figure 1D), were tested in K562 cells. As shown in Figure 2F, compound 7 showed $\mathrm{GI}_{50}$ of $0.114 \mu \mathrm{M}$ whereas compounds 13,14 and 15 were less active with $\mathrm{Gl}_{50}$ values of $3.85 \mu \mathrm{M}, 1.19 \mu \mathrm{M}$ and $0.384 \mu \mathrm{M}$, respectively. In an attempt to understand the loss of activity, the cell permeability of three compounds were tested (Tables S5 and S6); Compound 13 showed poor recovery rates which could be due to metabolism of the aldehyde functional group. ${ }^{[35]}$ The 10-fold improvement in the anti-proliferative activity of its ketone counterpart (e.g. 15; $\mathrm{GI}_{50}=0.384 \mu \mathrm{M}$ ) suggests that the aldehyde might not be the optimal choice for this scaffold in the cellular systems.

Finally, to evaluate the proteome-wide reactivity and potential off-targets of $\mathbf{1 4}$ in live mammalian cells, we synthesized its alkyne-containing analog (16; Scheme 
A<smiles>C#CCN(C)C(=O)c1cncc(-c2cnc3[nH]cc(-c4cc(C(=O)F)c(B(O)O)cc4OC)c3c2)c1</smiles>

16<smiles>C#CCNc1nc(Cl)nc(Cl)n1</smiles>

17

D

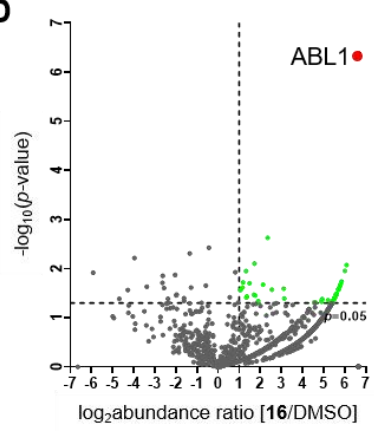

$\mathbf{F}$

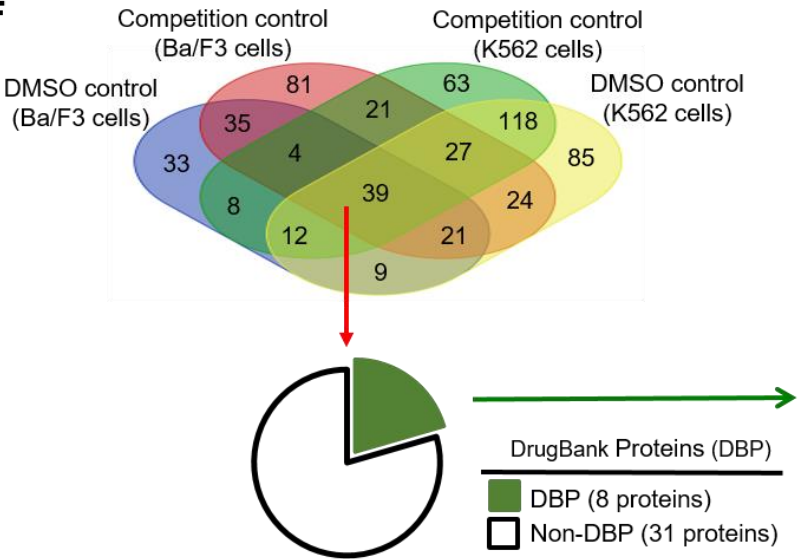

B
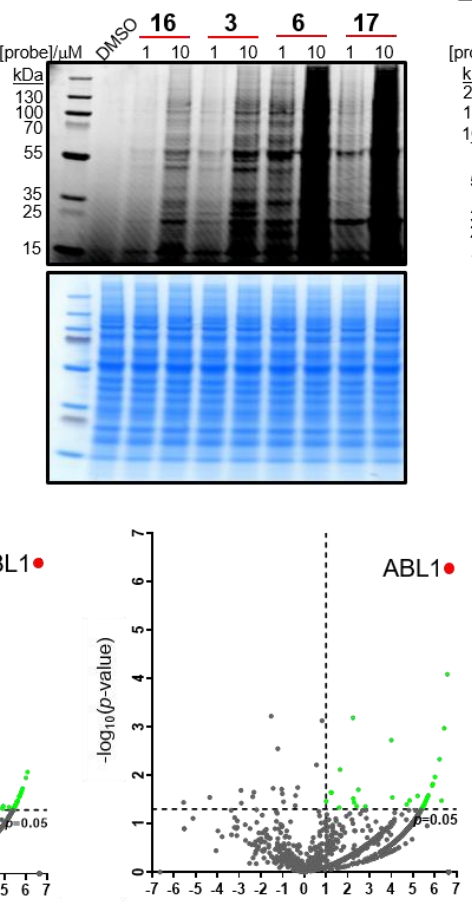
$\log _{2}$ abundance ratio [16/competition]

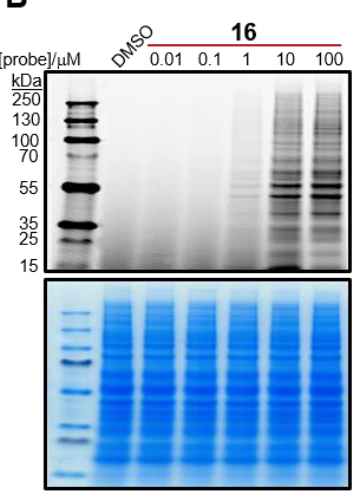

C $16-+++$

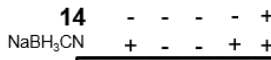
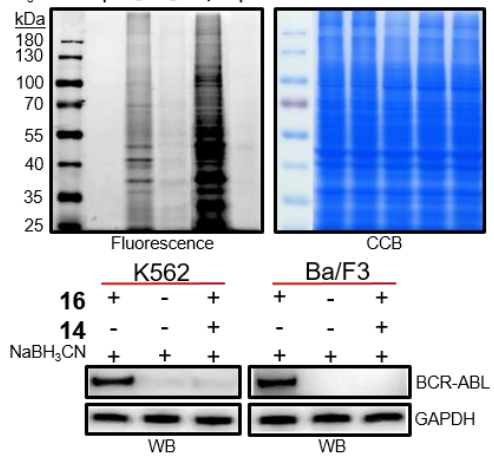

E
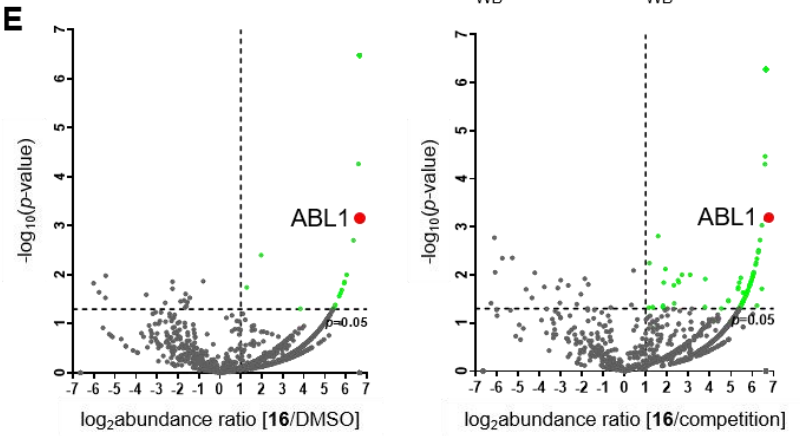

Functional classes of targets (39 proteins)

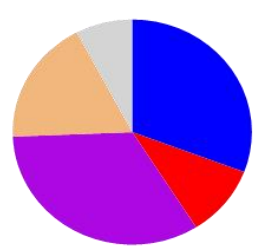

ABL1, PCMT1, ME2, ALDH1B1, RPIA SLC29A1

TPT1

CAPNS1

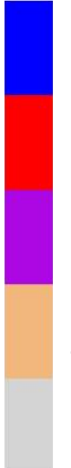

Enzymes

Transporters, channels and receptors

Gene expression and nucleic acid binding

Scaffolding, modulators and adaptors

Uncategorized

Figure 3. (A) Proteome reactivity profiles of compound $\mathbf{1 6}$ compared to $\mathbf{3}, \mathbf{6}$ and $\mathbf{1 7}$ in $\mathrm{K} 562$ cell lysates (in PBS with $0.1 \%$ Triton, $p \mathrm{H} 7.5$ ) (Top): in-gel fluorescence scanning; (Bottom): Coomassie staining (CBB). (B) Concentration-dependent proteomic reactivity profiles of 16 (0$100 \mu \mathrm{M}$ ) in $\mathrm{Ba} / \mathrm{F} 3$ cells overexpressing BCR-ABLWT. (C) Effect of washing by cold acetone and cold methanol of probe-labeled K562 (see Figure $\mathrm{S} 13$ for corresponding $\mathrm{Ba} / \mathrm{F} 3$ results) lysates in the absence and presence of excess $\mathrm{NaBH}_{3} \mathrm{CN}$ (top); Western blotting (WB) detection of BCR-ABL WT in lysates of both K562 and Ba/F3 cells following pull-down (PD) assays. GAPDH = loading control. (D/E) Volcano plots of potential cellular targets of 16 from probe-treated K562 and Ba/F3 cell lysates, respectively. (F) Data analysis of shared targets identified from chemoproteomic experiments in (D/E). See Figures S12, S13 and Table S7 for details.

1) and carried out large-scale chemoproteomic studies (Figure 3).[36] We first compared the proteome reactivity of the aldehyde-boronic acid moiety to other well-known lysine-targeting functionalities by using lysates from K562 cells (Figure 3A); [17,18] in-gel fluorescence scanning analysis of probe-labeled lysates, followed by CuAAC with rhodamine azide, ${ }^{[37]}$ showed that $\mathbf{3}$ demonstrated better selectivity at both $1 \mu \mathrm{M}$ and $10 \mu \mathrm{M}$ when compared to 6 and $\mathbf{1 7}$ which are known lysinereactive electrophiles, but was predictably less selective compared to the kinase-targeting 16, suggesting that molecular recognition was the dominant factor for similar compounds that contain low-reactivity electrophiles such as iminoboronates. ${ }^{[38,39]}$ In 16-treated samples, we observed a concentration-dependent labeling of proteomes, with saturated fluorescence signals at $\sim 10 \mu \mathrm{M}$ of the probe (Figure $3 \mathrm{~B}$ ). We next repeated the labeling experiment in the presence of $\mathrm{NaBH}_{3} \mathrm{CN}$ which helped trap the reversible covalent iminoboronates into more stable amine adducts (Figure 3C); a concomitant increase in the fluorescence intensity of the 16-labeled proteome was observed. In contrast, in the absence of $\mathrm{NaBH}_{3} \mathrm{CN}$, washing the 16labeled proteome with cold acetone and methanol significantly reduced or abolished the labeling, thus confirming the reversibility of iminoboronate bond 
(Figures S13 and S14). Upon further enrichment of the labeled proteomes by pull-down (PD) experiments followed by Western blotting (WB) analysis, we confirmed successful labeling of endogenous BCR-ABL from both $\mathrm{K} 562$ and $\mathrm{Ba} / \mathrm{F} 3$ cell lysates (Figure $3 \mathrm{C}$ bottom). By using DMSO-treated and 14-competed samples as controls, we carried out large-scale LC-MS analysis (Figures 3C-E, S11 and S12, Table S7).[22,36,37] Consistent with strong labeling shown by in-gel fluorescence scanning (Figure 3C), 16 captured a number of off-targets in addition to the expected BCR$A B L$ (highlighted in red in Figure 3D/E). Upon combining analyses of enriched hits from 16-labeled proteomes of both K562 and Ba/F3 cells, we identified a total of 39 potential cellular targets out of which 8 were DrugBank proteins including ABL1, PCMT1, ME2, ALDH1B1, RPIA, SLC29A1, TPT1 and CAPNS1 at a high confidence level (Figure $3 \mathrm{~F}$ and Table S7). Unlike protein kinases which have a known catalytic lysine residue in their kinase active site, most of these shared targets possess solvent-exposed lysine residues, rendering them susceptible to probe labeling. Interestingly, ABL1 was the only kinase successfully identified from our experiments in both cell lines, indicating 16 was a selective ABL-targeting probe towards kinases.

\section{Conclusion}

In summary, we have successfully demonstrated, for the first time, that the catalytic lysine of $A B L$ can be selectively targeted by inhibitors bearing the aldehyde boronic acid moiety, leading to a reversible covalent adduct. The incorporation of the two substituents required for covalent bond formation reduced the affinity to the $A B L$ active site; however, the slow formation of the iminoboronate led to highly potent inhibitors of $A B L$ kinase and its mutants. We also showed that the aldehyde boronic acid, which is a low-reactivity electrophile, can be used to design highly selective kinase inhibitors by maximizing molecular recognition. Such compounds might be attractive tools for chemical biology studies given recent interests in the development of reversible covalent inhibitors, ${ }^{[29,31,40]}$ but could also serve as potential drug candidates in cases where improved potency might be desirable once they are fully optimized.

\section{Acknowledgements}

Financial support was provided by the Synthetic Biology Research \& Development Programme (SBP) of National Research Foundation (SBP-P4 and SBP-P8) for Shao Q. Yao, the National Medical Research Council (NMRC) via the Open Fund - Young Individual Research Grant for Klement Foo and by the Agency for Science, Technology and Research (A*STAR) via the $A^{*}$ STAR Graduate Scholarship (AGS) for David Quach. Financial support from CAMS Innovation Fund for Medical Sciences (CIFMS) (2017-I2M-4-005) of China is also acknowledged. The ABL protein construct and glycerol stock were prepared by Yvonne Y. W. Tan and Dario B. Heymann.

Keywords: cancer $\bullet$ lysine $\cdot$ covalent $\cdot$ reversible $\cdot$ proteomics

[1] R. Kurzrock, J. U. Gutterman, M. Talpaz, N. Engl. J. Med. 1988, 319, 990-998.

[2] T. G. Lugo, A. M. Pendergast, A. J. Muller, O. N. Witte, Science 1990, 247, 1079-1082.

[3] E. Jabbour, H. Kantarjian, Am. J. Hematol. 2016, 91, 252-265.

[4] H. M. Kantarjian, M. Baccarani, E. Jabbour, G. Saglio, J. E. Cortes, Clin. Cancer Res. 2011, 17, 1674-1683.

[5] Z. Iqbal, A. Aleem, M. Iqbal, M. I. Naqvi, A. Gill, A. S. Taj, A Qayyum, N. ur-Rehman, A. M. Khalid, I. H. Shah, M. Khalid, R Haq, M. Khan, S. M. Baig, A. Jamil, M. N. Abbas, M. Absar, A. Mahmood, M. Rasool, T. Akhtar, PLos One 2013, 8, e55717.

[6] T. Anagnostou, M. R. Litzow, Blood Lymphat. Cancer 2017, 2018, 1-9.

[7] D. S. Johnson, E. Weerapana, B. F. Cravatt, Future Med. Chem. 2010, 2, 949-964.

[8] A. Chaikuad, P. Koch, S. A. Laufer, S. Knapp, Angew. Chem. Int. Ed. 2018, 57, 4372-4385.

[9] Z. Zhao, P. E. Bourne, Drug Discov. Today 2018, 23, 727-735.

[10] J. Engel, J. Lategahn, D. Rauh, ACS Med. Chem. Lett. 2016, 7, 2-5.

[11] S-H. I. Ou, Crit. Rev. Oncol. Hematol. 2012, 83, 407-421.

[12] Q. S. Liu, Y. Sabnis, Z. Zhao, T. H. Zhang, S. J. Buhrlage, L. H. Jones, N. S. Gray, Chem. Biol. 2013, 20, 146-159.

[13] S. Niessen, M. M. Dix, S. Barbas, Z. E. Potter, S. Lu, O. Brodsky, S. Planken, D. Behenna, C. Almaden, K. S. Gajiwala, K. Ryan, R. Ferre, M. R. Lazear, M. M. Hayward, J. C. Kath, B. F. Cravatt, Cell Chem. Bio. 2017, 24, 1388-1400.

[14] F. Rossari, F. Minutolo, E. Orciuolo, J. Hematol. Oncol. 2018, $11,1-14$.

[15] A. C. Carrera, K. Alexandrov, T. M. Roberts, Proc. Natl. Acad. Sci. U. S. A. 1993, 90, 442-446.

[16] R. Liu, Z. Yue, C. C. Tsai, J. Shen, J. Am. Chem. Soc. 2019 141, 6553-6560.

[17] D. A. Shannon, R. Banerjee, E. R. Webster, D. W. Bak, C. Wang, E. Weerapana, J. Am. Chem. Soc. 2014, 136, 3330-3333.

[18] C. C. Ward, J. L. Kleinman, D. K. Nomura, ACS Chem. Biol. 2017, 12, 1478-1483.

[19] A. Cuesta, J. Taunton, Annu. Rev. Biochem. 2019, 88, 365-381.

[20] M. Gehringer, S. A. Laufer, J. Med. Chem. 2019, 62, 5673-5724.

[21] S. M. Hacker, K. M. Backus, M. R. Lazear, S. Forli, B. E. Correia, B. F. Cravatt, Nat. Chem. 2017, 9, 1181-1190.

[22] Q. Zhao, X. Ouyang, X. Wan, K. S. Gajiwala, J. C. Kath, L. H. Jones, A. L. Burlingame, J. Taunton, J. Am. Chem. Soc. 2017, 139, 680-685.

[23] S. E. Dalton, L. Dittus, D. A. Thomas, M. A. Convery, J. Nunes, J. T. Bush, J. P. Evans, T. Werner, M. Bantscheff, J. A. Murphy, S. Campos, J. Chem. Am. Soc. 2018, 140, 932-939.

[24] U. P. Dahal, A. M. Gilbert, R. S. Obach, M. E. Flanagan, J. M. Chen, C. Garcia-Irizarry, J. T. Starr, B. Schuff, D. P. Uccello, J. A. Young, Med. Chem. Commun. 2016, 7, 864-872.

[25] J. Pettinger, K. Jones, M. D. Cheeseman, Angew. Chem. Int. Ed. 2017, 56, 15200-15209.

[26] P. Martín-Gago, C. A. Olsen, Angew. Chem. Int. Ed. 2019, 58, 957-966.

[27] L. H. Jones, Angew. Chem. Int. Ed. 2018, 57, 9220-9223.

[28] D. E. Mortenson, G. J. Brighty, L. Plate, G. Bare, W. Chen, S. Li, H. Wang, B. F. Cravatt, S. Forli, E. T. Powers, K. B. Sharpless, I. A. Wilson, J. W. Kelly, J. Am. Chem. Soc. 2018, 140, 200-210.

[29] I. M. Serafimova, M. A. Pufall, S. Krishnan, K. Duda, M. S. Cohen, R. L. Maglathlin, J. M. McFarland, R. M. Miller, M. Frödin, J. Taunton, Nat. Chem. Biol. 2012, 8, 471-476. 
[30] S. Cambray, J. Gao, Acc. Chem. Res. 2018, 51, 2198-2206.

[31] G. Akçay, M. A. Belmonte, B. Aquila, C. Chuaqui, A.W. Hird, M. L. Lamb, P. B. Rawlins, N. Su, S. Tentarelli, N. P. Grimster, Q. Su, Nat. Chem. Biol. 2016, 12, 931-936.

[32] T. Zhou, L. Parillon, F. Li, Y. Wang, J. Keats, S. Lamore, Q. Xu, W. Shakespeare, D. Dalgarno, X. Zhu, Chem. Biol. Drug Des. 2007, 70, 171-181.

[33] P. M. S. D. Cal, J. B. Vicente, E. Pires, A. V. Coelho, L. F. Veiros, C. Cordeiro, P. M. P. Gois, J. Am. Chem. Soc. 2012, 134 10299-10305.

[34] R. A. Bauer, Drug Discov. Today 2015, 20, 1061-1073.

[35] L. A. Admed, H. Younus, Drug Metab. Rev. 2019, 51, 42-64.

[36] B. R. Lanning, L. R. Whitby, M. M. Dix, J. Douhan, A. M. Gilbert, E. C. Hett, T. O. Johnson, C. Joslyn, J. C. Kath, S. Niessen, L. R. Roberts, M. E. Schnute, C. Wang, J. J. Hulce, B. Wei, L. O. Whiteley, M. M. Hayward, B. F. Cravatt, Nat. Chem. Biol. 2014, 10, 760-767.

[37] H. Shi, C.-J. Zhang, G. Y. J. Chen, S. Q. Yao, J. Am. Chem. Soc. 2012, 134, 3001-3014.

[38] K. M. Backus, B. E. Correia, K. M. Lum, S. Forli, B. D. Horning, G. E. González-Páez, S. Chatterjee, B. R. Lanning, J. R. Teijaro, A. J. Oison, D. W. Wolan, B. F. Cravatt, Nature 2016, 534, 570-574.

[39] S. Pan, S. Y. Jang, S. S. Liew, J. Fu, D. Wang, J. S. Lee, S. Q. Yao, Angew. Chem. Int. Ed. 2018, 57, 579-583.

[40] a) W.-H. Guo, X. Qi, X. Yu, Y. Liu, C.-I. Chung, F. Bai, X. Lin, D. Lu, L. Wang, J. Chen, L. H. Su, K. J. Nomie, F. Li, M. C. Wang X. Shu, J. N. Onuchic, J. A. Woyach, M. L. Wang, J. Wang, Nat Commun. 2020, 11, 4268; b) R. Gabizon, A. Shraga, P. Gehrtz, E. Livnah, Y. Shorer, N. Gurwicz, L. Avram, T. Unger, H. Aharoni, S. Albeck, A. Brandis, Z. Shulman, B.-Z. Katz, Y Herishanu, N. London, J. Am. Chem. Soc. 2020, 142, 1173411742. 


\section{Entry for the Table of Contents}

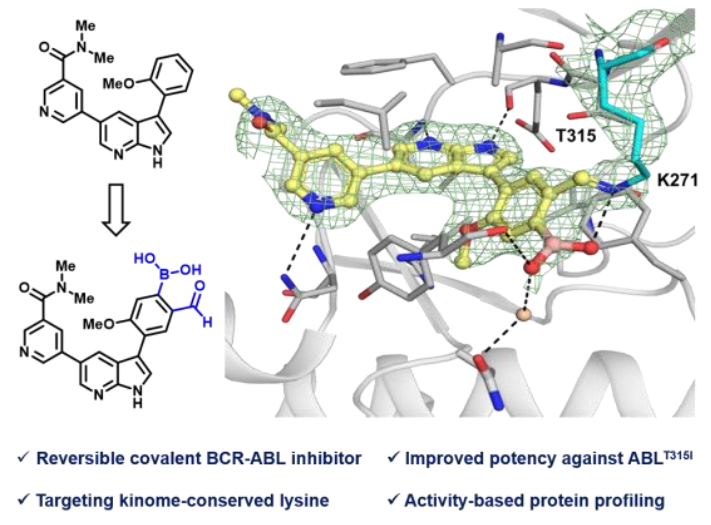

Using the iminoboronate chemistry, we report the first successful examples of catalytic lysine-targeting reversible covalent BCR$A B L$ inhibitors which inhibited both $A B L^{W T}$ and $A B L^{T 3151}$ at improved nanomolar potency. We also demonstrated for the first time how the study of off-targets for this class of compounds could be performed using activity-based protein profiling and proteomic mass spectrometry. 